\title{
MODELO PARA LA ORIENTACION DE TALENTOS EN FUTBOL SALA FEMENINO
}

Bucheli. J. ${ }^{1}$ Vera. J. ${ }^{2}$ Jaimes. M. ${ }^{3}$

1. Bucheli James ${ }^{1}$.Especialista en Actividad Física. andresbli17@hotmail.com

2. Vera José Luis 2. Doctor en ciencias de la cultura física, Profesor Universidad del Valle, Grupo de investigación Grinder. vera.jose@correounivalle.edu.co.

3. Marco Fredy Jaimes laguado ${ }^{3}$ Doctor en nuevas perspectivas de investigación en la actividad física, Profesor Universidad de Pamplona, Grupo de Investigación actividad Física y Deportes. mafrejala@hotmail.com.

\section{RESUMEN}

El presente trabajo investigativo tiene como finalidad crear un modelo para la orientación y selección de talentos deportivos en el futbol sala femenino y para ello se describe en el marco teórico una amplia referencia de conceptos de diferentes autores que han investigado sobre el tema a tratar, teniendo así una bibliografía especializada que permite una adecuada orientación. La investigación se plantea desde la perspectiva cuasi experimental, teniendo como población las niñas que hacen parte de la Institución Educativa la Planada de Balboa Cauca y una muestra de 15 niñas de entre 12 y 14 años, quienes hacen parte de la selección la cual representa en diferentes eventos deportivos y a quienes se le aplicaron pre test, de las acciones técnicas más importantes de la ofensiva en el futbol sala, después se someten a una intervención durante un periodo de cuatro meses para luego realizar post test y posteriormente analizar sus efectos y posibles cambios. Se concluye que la intervención presenta cambios significativos en todas las acciones técnicas además de las pruebas físicas y de fuerza explosiva, estas son tenidas en cuenta de acuerdo a las características específicas que tiene el deporte investigado, además la técnica de mayor evolución fue tiro a portería debido a que con ella se busca el principal objetivo que es el de anotar un gol, y la técnica de mayor complejidad en la ejecución y evolución es la conducción debido a que esta acción no es muy habitual en la vida cotidiana del genero investigado.

Palabras clave: Orientación, talento deportivo, futbol sala, acciones técnicas

\begin{abstract}
This research work aims to create a model for orientation and selection of sports talents in female football and for this it's describe reference to concepts of different authors who have researched the issue to be addressed is described in the theoretical framework, taking and a specialized bibliography that allows proper guidance. The investigation arises from the quasi-experimental perspective, with the population girls who are part of the educational institution Planada de Balboa Cauca and a sample of 15 girls between 12 and 14 years, who are part of the selection which represents different sporting events and those were applied pre test, the most important technical actions of the offensive in futsal, then subjected to an intervention during a period of four months and then perform post test and then analyze their effects and possible changes. We conclude that the intervention has significant changes in all technical actions in addition to the physical and explosive strength tests, these are taken into account according to the specific characteristics of sport investigated, besides the technique further evolution was the shot at goal because this is looking the main objective is to score a goal, and the technique of greater complexity in the implementation and evolution is driving because this action is not very common in everyday life of the genre investigated.
\end{abstract}

Keyword: Orientation, athletic talent, futsal, technical actions

\section{Convocatoria III. Trimestre. Recibido el 15 de junio de 2017; Aceptado el 15 de julio de 2017.}




\section{INTRODUCCION}

Crear una propuesta de modelo para la orientación de talentos más específicamente en el futbol sala femenino, el cual permita dar una serie de parámetros a tener en cuenta a la hora de seleccionar ya que implicara un análisis mucho más detallado del perfil que debería tener las interesadas en actuar para dicho deporte, el cual servirá como referencia para todos los involucrados en el futbol sala femenino, es la intención del presente texto y para ello se ha investigado con diferentes autores que platean su poción frente al tema a tratar.

Los métodos y los medios de detección y posterior selección que se utilizan, en muchos casos no son lo suficientemente rigurosos y exactos porque no están basados en la autenticidad estadística y científica, o se han realizado de forma rutinaria, no comprendiendo todas las facetas que intervienen en la preparación y rendimiento de un deportista que ha de competir a altos niveles de esfuerzo. (Álvarez Villar, 1984).

Modelos procesuales donde se trata de la detección científica estableciendo diversas tapas para la predicción definitiva de los talentos deportivos lo que evitaría los problemas que acarrean las detecciones puntuales y evitan según Gimbel, B. (1976) "los falsos positivos", es decir, los que han sido identificados como "dotados", pero que no alcanzan la cima de su especialidad

La detección de talentos representa un elemento propio de la organización deportiva, lo cual permite interpretar la detección de talentos como un proceso sistemático, planeado a medio y largo plazo. (Bompa, T. (1985)

La formación deportiva ha de basarse en una educación polivalente multilateral he integra, de manera que desarrollen todos los ámbitos de la persona: motor social afectivo y cognitivo Debe ser un trabajo constante de mucha dedicación, paciencia llevando un proceso acorde a las necesidades con un orden cronológico y de la mano de un grupo de personas especializadas en el tema además del núcleo que rodea al deportista. (Giménez, 1999).
En los países del este de Europa la clave de este está en la inclinación a la selección precoz de talentos, se diferencian en unos en el desarrollo somático de los jóvenes, otros analizan aspectos relacionados con la medicina del deporte, otros toman en cuenta las características psicológicas como base de la selección de jóvenes.

España es uno de los modelos más destacados en la formulación de objetivos acorde a la edad de los jugadores y en progresión con respecto a la etapa anterior, contenidos de trabajos afines a los objetivos propuestos y unas orientaciones para la enseñanza aprendizaje dirigidas al desarrollo de los contenidos de trabajo. (Palau, 2009)

Es quizás el modelo español uno de los mejores si tenemos en cuenta los resultados deportivos que estos han arrojado siendo campeones mundiales en el futbol sala y que genera en este país gran acogida por parte de su población, además de contar con una de las ligas de mayor prestigio a nivel mundial donde los jugadores profesionales buscan llegar a esta y por ende es una referencia importante a tener en cuenta en el momento de investigar.

Ahora bien, en nuestro país los modelos están basados en la experiencia que ha adquirido el personal encargado de la selección de talentos, además de quienes a través de las diferentes competencias organizadas en cada uno de los departamentos se encargan de recopilar los talentos con los cuales se inicia un trabajo de preparación para que posteriormente sean quienes representes en diferentes eventos tanto nacionales como internacionales.

En el departamento del Cauca el futbol sala femenino ha surgido por intermedio de programas que tan solo a nivel universitario han tomado fuerza ya que tienen competencias mucho más organizadas como lo son los juegos universitarios con la gran diferencia que las deportistas a esta edad en su mayoría no han tenido un proceso de maduración futbolística y que sus condiciones las han adquirido a través de un juego empírico y que es en el proceso de formación académica donde logran obtener una mejor condición tanto física, técnica como táctica. 
Es por ello que por intermedio de la presente investigación se quiere dar a conocer una propuesta de modelo para la orientación de talentos más específicamente en el futbol sala femenino, el cual permita dar una serie de parámetros a tener en cuenta a la hora de seleccionar ya que implicara un análisis mucho más detallado del perfil que debería tener las interesadas en practicar dicho deporte, el cual servirá como referencia para todos los involucrados en el futbol sala femenino teniendo en cuenta que pocos los estudios en modelos que se han realizado teniendo como muestra un población de género femenino mucho menos con el género femenino.

\section{MARCO METODOLOGICO}

\section{MÉTODO}

Para la presente investigación se diseñó pre test y pos test para un solo grupo ya que la variable dependiente es medida antes y después de la manipulación de la variable independiente y posteriormente se computa la magnitud del cambio, si es que este se produce. En este caso solo se emplea al grupo de sujetos experimental, que no es seleccionado al azar y que se utiliza además como su propio control.

La institución Educativa la Planada, es el lugar donde se desarrolla la investigación, teniendo como población 263 estudiantes inscritos y se realiza una muestra con 15 niñas de 12 a 14 años de edad que hacen parte de la selección que representa a la Institución de diferentes eventos deportivos.

Se evalúa a través de la siguiente batería de test las técnicas de conducción, tiro a portería, recepción pase, además de toma antropométrica, fuerza explosiva y resistencia, para luego realizar una intervención durante cuatro meses con una intensidad de 3 veces por semana y una duración de 90 minutos por unidad de entrenamiento, terminado este proceso se procederá de nuevo a evaluar y analizar sus posibles cambios.

\section{El diseño de pruebas e instrumentos del modelo}

Test de de Course Navette (leger)
Objetivo: Determinar la potencia aeróbica máxima. Determinar el VO2 máximo.

Al iniciar la señal el atleta deberá correr hasta la línea contraria (20 metros), pisarla y esperar escuchar la segunda señal para volver a desplazarse, el deportista debe intentar seguir el ritmo que marca el magnetófono que progresivamente irá incrementando el ritmo de carrera. El test finalizará en el momento que el ejecutor no pueda pisar la línea en el momento que lo marque el magnetófono.

A cada período rítmico lo denominaremos "palier" o "período" y tiene una duración de 1 minuto, los resultados se pueden determinar en la correspondiente tabla de baremación. Calculo del Volumen Máximo de Oxigeno VO2 máximo $=5,857 \times$ Velocidad $(\mathrm{Km} / \mathrm{h})-19,458$

Normas: Se deberá pisar la línea señalada en cada uno de los desplazamientos, de lo contrario la prueba debe ser cancelada. El atleta no podrá pisar la siguiente línea sin escuchar antes la señal del magnetófono, la cual se va incrementando a medida que los períodos aumentan. Cuando el atleta se vea imposibilitado a seguir el ritmo del magnetófono, finalizará la prueba y se anotará el último período o mitad de período escuchado.

Material: Pista 20 metros de ancho, magnetófono y cassette con la grabación del protocolo del Test de Course Navette.

Grafico descriptivo:

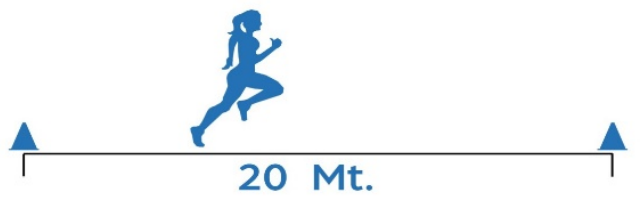

Fuente: Sebastián Salgado 2016

Test Sargent

Objetivo: Medir la fuerza explosiva de la musculatura de los miembros inferiores. 
Para este test se debe realizar un salto sin impulso en dos pies, desde un punto inicial y caer en los mismos dos pies (sin arrastrarlos 0 moverlos) y medir la distancia desde el talón que quede más cercano al punto de partida, ese es el resultado.

Grafico descriptivo:

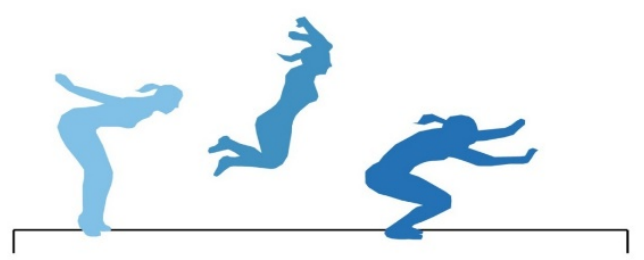

Fuente: Sebastián Salgado 2016

Para las siguientes valoraciones se tiene como referencia la batería de test propuesta por Roberto Montes García en el 2006

Test Tiro a Portería.

Objetivo: Identificar la efectividad del tiro a portería

\section{Descripción metodológica:}

El test comienza cuando el jugador amarillo realiza un pase al pie al jugador azul, el cual debe realizar un tiro a portería desde fuera del área antes de que el jugador verde (que empieza a correr en el momento en que el jugador amarillo realiza el pase), le alcance e intercepte el tiro.

Cada jugador realizará 5 tiros recibiendo el pase desde la derecha como se explica en el dibujo anterior, y 5 tiros recibiendo el pase por la izquierda, saliendo el defensor desde el lado contrario.

El jugador azul (el cuál es evaluado), debe de conseguir realizar el tiro de forma rápida, de manera que logre golpear al balón sin que el jugador le moleste, por lo que esta será la primera valoración que hagamos del jugador (velocidad de tiro).

Para evaluar los otros dos parámetros (precisión y potencia), hemos colocado un objetivo en el interior de la portería (4 conos, 2 junto a cada poste), los cuáles los jugadores deben intentar derribar, de tal forma que se combine en el golpeo a portería tanto la precisión (tocar el cono), como la potencia (derribar el cono).

\section{Grafico descriptivo:}

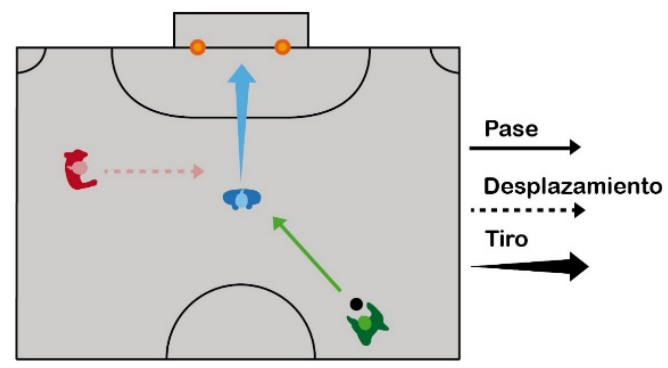

Fuente: Sebastián Salgado 2016

Test Pase.

Objetivo: Identificar la efectividad y precisión del pase

Descripción metodológica:

En banda, y a la altura del medio del campo, se sitúan tanto el jugador fucsia, como el jugador el azul, este último con balón. Un poco más retrasado y enfrente del palo derecho de la portería se sitúa el jugador verde (al cual se la valorará la cualificación técnica en el pase), y por último se colocarán dos conos a mitad de camino entre el jugador verde y el córner de banda izquierda, y otro cono 2 metros detrás de la línea de medio campo.

El test comienza cuando el jugador azul realiza un pase al jugador verde, el cuál tras controlar el balón deberá devolvérselo realizando un pase, para que éste reciba el balón y dispare a portería. En el momento que comienza el test, el jugador fucsia debe de dirigirse al cono que está detrás de él y tras tocarlo, intentar evitar que el jugador azul realice un disparo a portería

El jugador verde, debe intentar tener una buena precisión espacial en el pase intentando golpear el balón de tal manera de que este pase entre los dos conos colocados previamente, y a la vez tener una buena precisión temporal, adecuándose el pase a la trayectoria de carrera de su compañero, de forma que si realiza un buen pase, el jugador azul podrá realizar un 
disparo sin ninguna presión por parte del defensor.

Grafico descriptivo

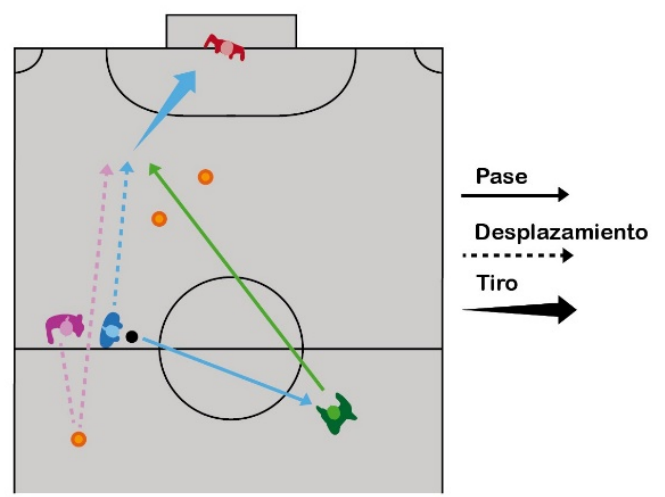

Fuente: Sebastián Salgado 2016

Test Conducción.

Objetivo: Identificar la manera de conducir el balón sobrepasando determinados obstáculos

Descripción metodológica:

Los dos conos naranjas colocados en el córner, será la salida del circuito de conducción. Se colocarán también los siguientes conos:

En la línea de área a medio camino entre la línea de banda derecha y el palo corto se colocará un cono (marrón). Lo mismo se hará también en la parte izquierda, colocando otro cono (morado). Un metro detrás de la portería, se colocará otro cono (fucsia). En el córner izquierdo, se colocará un cono (rojo).

Por último se coloca un cono (verde) a un metro del córner izquierdo (justamente donde comienza la línea del área).

El jugador, deberá hacer el siguiente recorrido, primeramente sin balón y posteriormente, tras un descanso con balón:

Sale en el córner izquierdo entre los conos naranjas, dirigiéndose al cono naranja, el cuál rodea, luego va hacia el cono fucsia de detrás de la portería, desde ahí pasará al córner izquierdo (cono rojo), el cual también rodea para luego dirigirse al cono morado, desde el cono morado irá a dar la vuelta al cono fucsia, para volver hacia el cono verde, al cual lo rodeará por fuera, para luego correr por fuera de la línea de área hacia los conos naranjas de la salida, rodeándolos y entrando a la llegada por detrás de ellos.

Primeramente se anotará el tiempo, que el jugador tarda en realizar el recorrido sin balón, para luego compararlo con el tiempo que tarda en realizar el circuito con balón, de esta forma cuanto menos diferencia exista entre ambos tiempos mayor será la cualificación técnica del futbolista en la conducción, ya que el balón se habrá llevado cerca del pie, y sin que se pierda mucha velocidad en la carrera, es decir, de forma más natural.

$\%=$ (Tiempo de conducción sin balón / t. con balón) $\times 100$

\section{Grafico descriptivo}

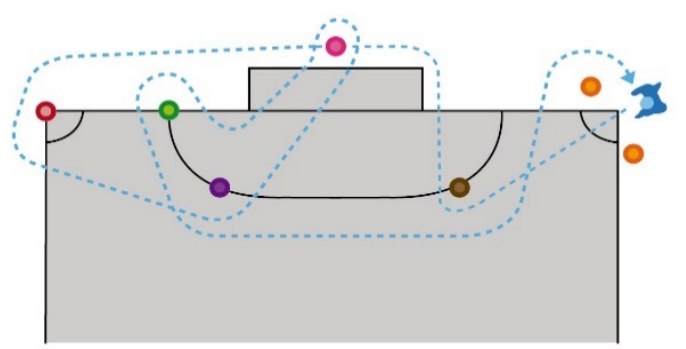

Fuente: Sebastián Salgado 2016

De manera que cuanto más cercano sea el porcentaje al $100 \%$, mayor será la cualificación técnica del deportista en la conducción de balón.

Test Control.

Objetivo: Identificar la capacidad de controlar el balón

Descripción metodológica:

El jugador marrón (el cuál será evaluado en este test de control de balón) se situará al borde del área, de cara al jugador azul, que se encuentra en el medio del campo con el balón. Detrás del jugador marrón se situará un defensor, y en la portería un portero, que aunque no intervenga en el test, si nos servirá para dar una mayor 
contextualización al test, así como una mayor motivación a los jugadores al enlazar el control con una acción posterior que será un tiro a portería. A la altura de la línea de puntos se situarán 2 conos (uno frente a cada poste de la portería).

El test lo comenzará el jugador azul, el cual realizará un pase al jugador marrón, que deberá controlar el balón y realizar un disparo a portería. En el momento que el jugador azul realiza el pase, el defensor, debe correr a tocar cualquiera de los dos conos situados en la línea de puntos, y una vez lo toque deberá intentar evitar que el jugador marrón no logre tirar a portería.

El jugador marrón debe de anticiparse al balón, para así evitar posibles anticipaciones defensivas, y además debe de fijarse en el movimiento del defensor, y de esta manera orientar el control de balón hacia el lado contrario de donde esté éste. También ha de utilizar las superficies de contacto adecuadas para la situación (interior del pie), y utilizar la pierna adecuada para controlar el balón y no sólo la dominante, ya que en algunos casos esto puede endentecer el control. Si el jugador cumple todo esto conseguirá realizar un tiro a portería antes de la llegada del defensor.

\section{Grafica descriptiva}

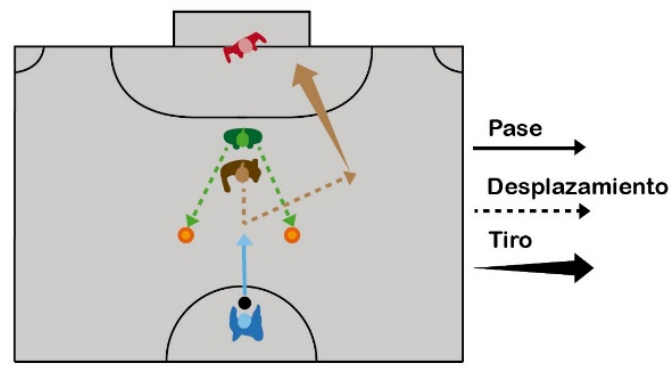

Fuente: Sebastián Salgado 2016

\section{Medidas antropométricas}

son: el peso corporal, estatura, medidas trasversales 0 diámetros en las que se encuentran diámetro de rodilla, diámetro de muñeca, diámetro de codo, también las medidas circunferenciales o perímetros tomando como principales cintura, cadera, antebrazo, brazo relajado, brazo contraído, muslo medio, pantorrilla además de los pliegues cutáneos dando como principales tríceps, subescapular, bíceps, axilar, pectoral, supra iliaco, supraespinal, abdominal, muslo anterior, muslo posterior, pantorrilla medial. (Alba, A. 2010)

\section{RESULTADOS}

Tendremos en cuenta que para la presente investigación se realiza un pre test, luego una intervención durante un periodo de cuatro meses para finalizar con la toma de pos test teniendo como referencia a Roberto Montes García con su batería de test para la valoración de la cualificación técnico-táctica de los futbolistas de futbol sala, categoría Benjamín, además de una valoración antropométrica, fuerza explosiva y de resistencia las cuales se consideran necesarias debido a las características propias que presenta el futbol sala.

\section{Leger pre test y post test}

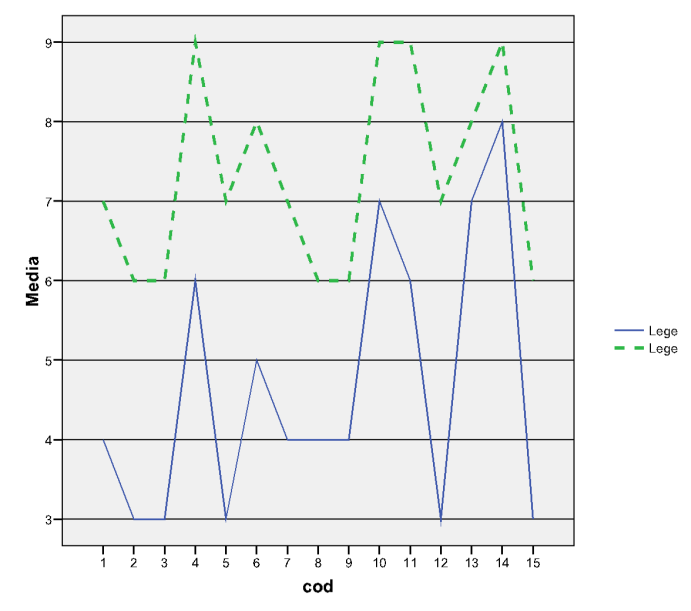

Fuente: Mg. Yaneth Molano 2015

De acuerdo a los resultados obtenidos en el pre test la gráfica nos indica que solo cuatro de las deportistas realizaron 6 minutos 0 más indicando de esta forma que el grupo se encuentra con una potencia aeróbica máxima en un tiempo de 4,66 minutos en promedio y que solo una deportista alcanza los 8 minutos, para el post test todas alcanzan y superan los 6 minutos en la prueba e incluso cinco de ellas 
alcanzan o pasan los 8 minutos demostrando así la mejoría en resistencia física con la intervención dada durante los cuatro meses

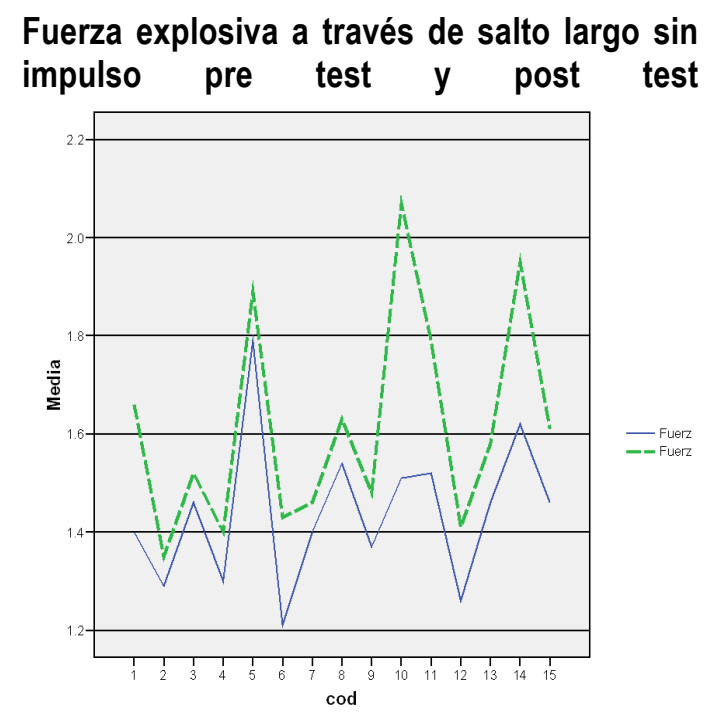

Fuente: Mg. Yaneth Molano 2015

Teniendo en cuenta las características propias del futbol sala como la explosividad es un test donde nos muestra que en promedio inicial esta en 1,43 metros y que en post test es 1,61 metros, en pre test seis deportistas dan resultados inferiores al 1,4 metros y que solo una alcanza la barrera de los 1,8 metros. En el pos test solo una deportista está por debajo de los 1,4 metros y tres ya superan los 1,8 metros, demostrando de esta manera que el tiempo de la intervención fue favorable y por ende la mejoría en los resultados pasando de un rango muy bajo a normal y que tres de las deportistas en el post test ya se encuentran en el mejor rango que es muy bueno.

\section{Pre test y post test de tiro a portería}

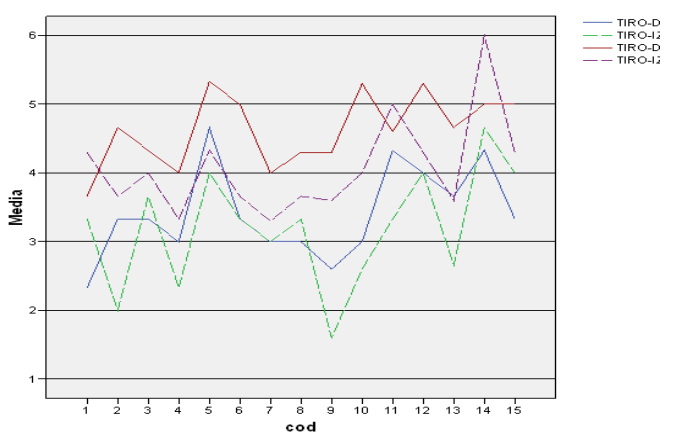

Fuente: Mg. Yaneth Molano 2015

Teniendo en cuenta la gráfica identificamos que la media del grupo se encuentra entre los 3 y 4 puntos de acuerdo a la valoración establecida, que en el pre test con el pie derecho la puntuación más baja fue de 2,33 y con el izquierdo de es de 1,6 además el tiro a portería en pos test de pie derecho se encuentra por encima de la media y que el mejor puntaje obtenido es de 5,33 y con el pie izquierdo es de 6 puntos lo que nos indica una gran mejoría con respecto al tiro a portería siendo esta técnica clave en el buen desarrollo de la práctica de este deporte

Pre test y post test de pase

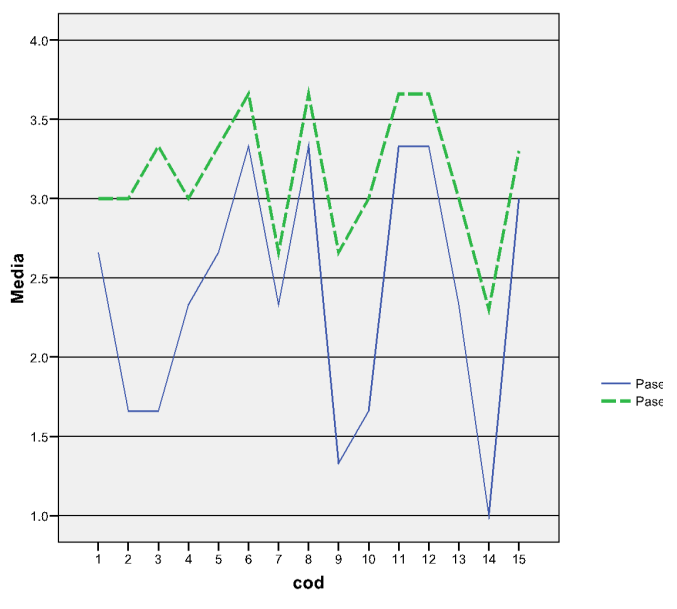

Fuente: Mg. Yaneth Molano 2015

Teniendo en cuenta la gráfica para la técnica de pase se identifica que se encuentra en una media de 2,5 puntos, que en el pre test solo 5 
deportistas logran estar por encima de dicho número, mientras que en el post test solo una deportista se encuentra por debajo del promedio, demostrando de esta manera que es una de las técnicas de mayor evolución del grupo dentro del proceso de intervención.

\section{Pre test y post test de conducción}

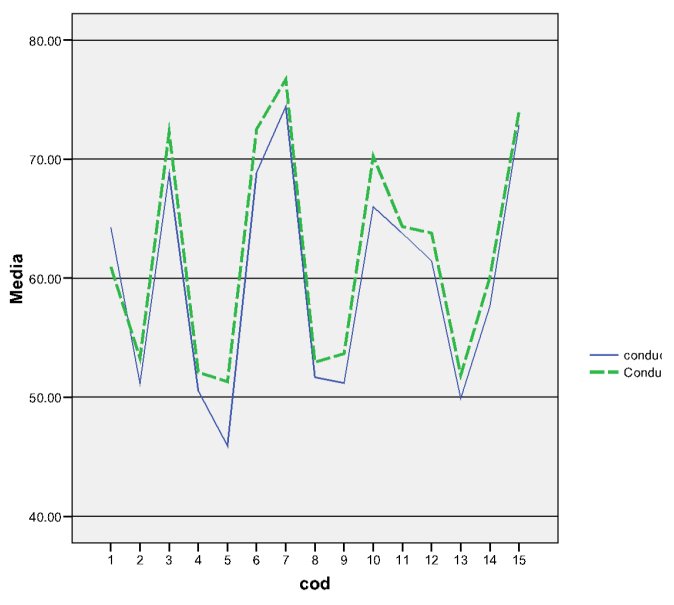

Fuente: Mg. Yaneth Molano 2015

En el análisis para la técnica de conducción teniendo en cuenta la gráfica se puede decir que no se presenta una gran diferencia en los datos arrojados por el pre test y post test a lo que se le puede atribuir por dos motivos, el primero que teniendo como población el género femenino sus condiciones son un poco más complejas al momento de la realización de dicha técnica y la segunda es que las características propias del futbol sala moderno hace que se juegue mucho más intenso y rápido lo que genera muy poco traslado del balón con los pies y si un mayor despliegue físico y precisión lo que no quiere decir que se encuentren en un nivel bajo ya que la media indica un $60 \%$ de efectividad destacándose dos deportistas que se encuentran por encima del $70 \%$ en el pre test y aumentando a cinco en el pos test

Pre test y post test de control

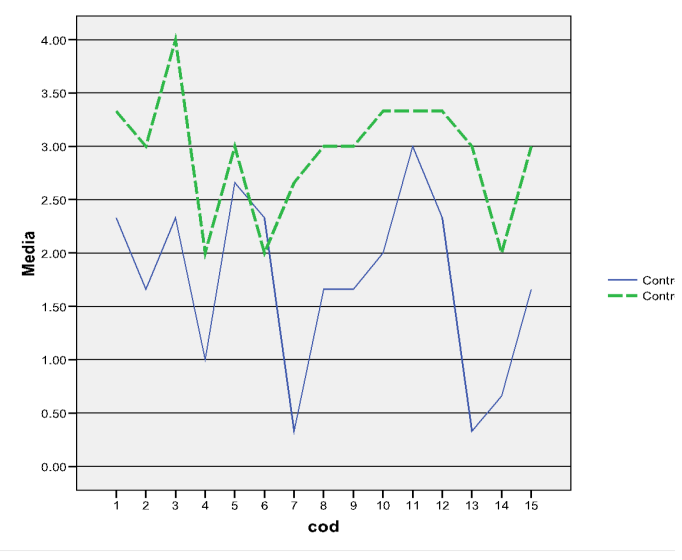

Fuente: Mg. Yaneth Molano 2015

La grafica para el test de control nos indica que en el pre test las deportistas evaluadas nueve de ellas no superan los 2 puntos, y que tan solo una deportista alcanza la valoración de 3 puntos, tomando como referencia que la media del grupo es de dos como lo indica la gráfica y que para la valoración después de realizar la intervención en el post test solo tres deportistas indican tener una valoración de dos y las demás superan esta barrera inclusive alcanzando los tres puntos

Análisis antropométrico

Porcentaje de grasa

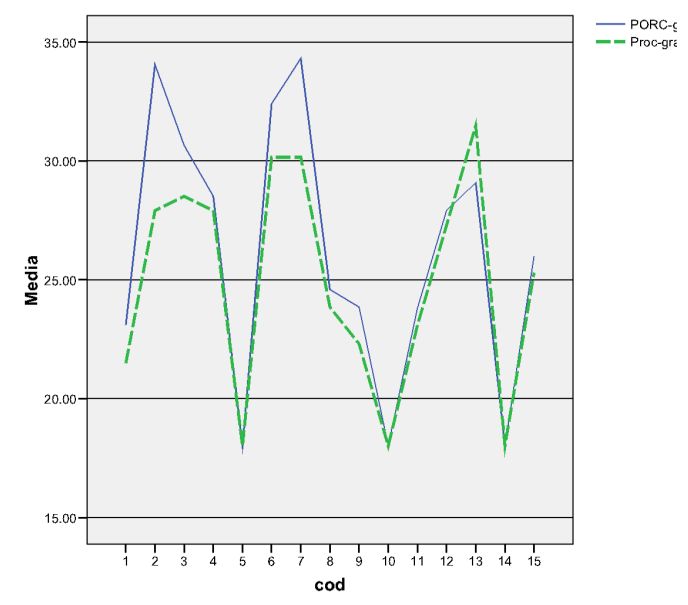

Fuente: Mg. Yaneth Molano 2015

Los datos que nos indican en el porcentaje de grasa muestran que la media se encuentra en 
25 que en el pre test el porcentaje mayor fue de 34,31 y el más bajo se encontró en 18,01, mientras que en el pos test el porcentaje más alto estuvo en 33 y el más bajo en 16 y que tres de las deportistas están por debajo de la barrera de los 20 lo que nos indicaría que se encuentran en un rango de normal teniendo en cuenta la edad y el género aunque con la continuidad de la práctica deportiva este debería llegar por lo menos al rango de bueno.

Indice de masa corporal

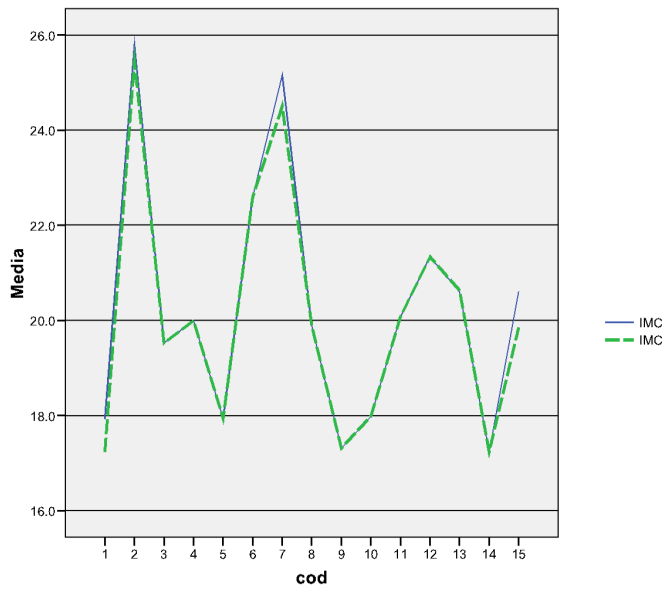

Fuente: Mg. Yaneth Molano 2015

El dato menor en el índice de masa corporal para el pre test nos indica que se encuentra en 17,2 que indica una delgadez grado I y el mayor en 25,7 que estaría en sobrepeso y para el pos test es de 16 que es delgadez grado I el menor y de 24 el mayor, que indica un grado normal para lo cual se debe tener en cuenta la talla y el peso y que en promedio grupal en pre test se encontraba en sobrepeso y en pos test normal

\section{DISCUSION}

La implementación de la propuesta metodológica para diseñar el modelo de orientación y selección de talentos a través de las acciones técnicas más importantes de la ofensiva en la selección del futbol sala en las niñas de 12 a 14 años de la Institución Educativa la Planada en el municipio de Balboa - Cauca, teniendo en cuenta los resultados obtenidos demuestra que la aplicación de pruebas técnicas permite ayudar a identificar talentos deportivos y que además se contribuye en la investigación de género femenino.

Alfaro (2004) plantea que "en la detección y desarrollo de las personas con altas capacidades motrices, la mujer tiene menos oportunidades que el hombre que se encuentra en sus mismas condiciones, por lo que sería necesario concientizar a los profesores, entrenadores y a las familias en este sentido para que favoreciesen la práctica deportiva de las chicas y para que faciliten la detección temprana de altas capacidades en las jóvenes practicantes".

Se valora la resistencia a través del test de leger el cual permite identificar la condición física del evaluado y para ello Edwards, Clark, \& Macfadyen (2003), plantea que "el consumo máximo de oxígeno (VO2 max) es un indicador útil para la estimación de la capacidad aeróbica, para determinar el nivel de entrenamiento y para la pla-nificación del mismo" teniendo en cuenta que la metodología utilizada para buscar una mejoría en la condición física de las deportistas evaluadas indico que se tuvo una mejoría individual y por ende colectiva (Bangsbo, 2002) plantea que "la condición física es uno de los factores que determina la capacidad del deportista de rendir durante una competición. La capacidad física individual contribuye a mejorar el rendimiento colectivo en los deportes de equipo".

Desde el punto de vista de la fuerza explosiva a través del test de salto largo sin impulso se puede ver que se genera una gran evolución en los resultados de pre test a post test teniendo en cuenta la intervención que se realiza en la investigación y para ello Palau (2007) indica que "a partir de los 10 años hasta los 12 aumenta en especial la fuerza rápida y sigue su evolución hasta los 14 años de forma conjunta con la fuerza explosiva, periodo en el cual empieza a tener un peso específico el valor de la fuerza muscular"

Teniendo en cuenta las características propias en la práctica del futbol sala y desde la resistencia, autores como Díaz y García (2002) plantean que los esfuerzos suelen ser de intensidad máxima y sub-máxima, localizados en el tren inferior y que la duración de los 
esfuerzos intensos continuados puede oscilar entre los 2 y 6 segundos, siendo estos tiempos tenidos en cuenta en las diferentes unidades de entrenamiento en la intervención realizada y en la valoración de pre test y post test con salto largo sin impulso.

Los resultados obtenidos después de realizar la intervención demuestran una mejoría en la técnica de conducción desde el punto de vista de la velocidad en el manejo del balón siendo este de los más complejos debido a varios factores que influyen directa e indirectamente en este proceso y para lo cual Fernández, Sánchez y Fernández (2002) plantean que "si se analiza la actividad del futbol, esta, se destaca por una gran importancia de los aspectos coordinativos en el manejo del balón con el pie, además de la coordinación óculo - pedica, y su utilización táctica en situaciones de juego debido a la presencia de compañeros y adversarios. Además de lo mencionado por el autor el género investigado también es un ítem especial a tener en cuenta para el adecuado desarrollo de dicha técnica, otros autores como Gómez y col (2007) citan a Wilmore (1982) donde plantea que dicho tratamiento debe tenerse en cuenta de acuerdo a las diferencias por género en el aprendizaje de las actividades físico deportivas ya sea por aspectos cuantitativos del movimiento, tiempo de práctica, motivaciones, autoestima.

Se destaca la valoración con la que se termina el proceso realizando el post test de las diferentes técnicas demostrando una mejoría en su mayoría notoria y autores como Morales quien cita a Pansza (2004) el cual propone que "un proceso continuo que permite juzgar el logro de los objetivos propuestos, aporta datos valorativos que permiten tomar decisiones para realizar los ajustes pertinentes"

\section{CONCLUSIONES}

El modelo planteado es estadísticamente apropiado para la detección de talentos en futbol sala femenino ya que la aplicación metodológica a través de las unidades de entrenamiento presentan un incremento en sus resultados en el análisis de pre test con post test. La elaboración de un modelo para la orientación de talentos deportivos debe estar en continua evolución lo que genera estar actualizando su estado y por ende su metodología de aplicación

Las unidades de entrenamiento bien planificadas genera en la deportista un mayor interés por la práctica del futbol sala y con ello continuidad y motivación en el proceso del perfeccionamiento de las diferentes acciones técnicas. Las pruebas y test son una herramienta útil en el proceso de identificar talentos lo más adecuado posible contribuyendo significativamente a la objetividad y efectividad.

\section{REFERENCIAS BIBLIOGRÁFICAS}

Alba, L. (2010) Test funcionales cineantropometria y prescripción del entrenamiento en el deporte y la actividad física p 150-169 editorial Kinesis.

Alfaro, E. (2004) El talento psicomotor y las mujeres en el deporte de alta competición. Revista de educación, 335, p. 146.

Álvarez, J. López, I. Echavarría, J. (2009) Análisis científico de diferentes métodos de entrenamiento en el futbol sala. Archivos de medicina del deporte. XXVI (130), p. 93-103.

Bangsbo, J. (2002). Entrenamiento de la condición física en el fútbol. Barcelona: Paidotribo.

Barbero, J.C. (2003) Análisis cuantitativo de la dimensión temporal durante la competición en futbol sala. Revista motricidad, 10, p. 143-163.

Cortez, J, (2014) Fundamentos tácticosestratégicos del futbol de salón. Revista de Educación Física, 3. Universidad de Antioquia, Instituto Universitario de Educación Física.

Díaz, J.A., García, J.J. (2002). Preparación física en alta competición. Madrid: Federación Madrileña de Futbol Sala.

Edwards, A., Clark, N., \& Macfadyen, A. (2003). Lactate and venti-latory thresholds reflect the training status of professional soccer pla-yers where maximum aerobic power is unchange. Journal of Sports Science and Medicine, 2, 2329. 
Fernández, E; Sánchez, F, y Fernández, C. (2002). Estudios de las habilidades de resolución de problemas en el futbol sala desde una perspectiva de género. Editorial, Estudios sobre ciencia del deporte. Mujeres y actividad física deportiva. 35. Madrid: MEC y D.CSD.

García, J.M.; Navarro, M y Ruiz, J.A. (1996) Planificación del entrenamiento deportivo. Madrid, Gymnos.

Giménez, F. J. (1999). Fases en la formación del deportista y su aplicación en la iniciación deportiva. Habilidad Motriz (15), 35-39.

Gómez, M; Ortega, E; Sainz, P. (2007). Diferencias en la ejecución técnicas en el futbol: análisis por género y nivel de experiencia en educación física. Federación española de Asociaciones de Docentes de Educación Física.

Irurtia, A. Iglesias, X (2010) La formación de los jóvenes deportistas: de la detección del talento a la intervención federativa. Instituto Nacional de Educación Física de Cataluña. Universidad de Barcelona.

López Bedoya, J. (1995) Entrenamiento temprano y captación de talentos en el deporte. Barcelona, Inde, 207-247

Montes García, R. (2006) Batería de test para la valoración de la cualificación técnico-táctica de los futbolistas de futbol sala, categoría Benjamín. Buenos Aires. Disponible en efedeportes.com /Revista digital. Consultada en enero 7 de 2015

Morales, G, (2004). Estrategia de enseñanza aprendizaje táctico en futbol sala (primera parte). Cuba.

Palau, X. (2007). Los multisaltos: ejercicios de fuerza aplicados al futbol sala. 113. Buenos Aires. Disponible en efedeportes.com /Revista digital.

Palau, X. (2009). Escuela de fútbol sala (V). Propuesta de objetivos y contenidos en la etapa de rendimiento (juveniles).

Rodrigo, M. (2010) La formación técnica del jugador de futbol sala desde las edades tempranas, el entrenamiento de la técnica adecuado a cada edad. Madrid.

Ruiz, L.M. y Sánchez, F. (1997) Rendimiento deportivo: claves para la optimización del aprendizaje. Madrid, Gymnos.

Silva, R. (2001) Entrenamiento con ejercicios intermitentes para mejorar el rendimiento deportivo en deportistas juveniles de futbol de salón de la ciudad de Pamplona. Revista Actividad Física y Desarrollo Humano, 2, p. 74 84

Tolosa, J. Mariño, N. (2013) Efecto de dos métodos de entrenamiento sobre la flexibilidad de deportistas de futbol sala. Revista Actividad Física y Desarrollo humano, p. 151 - 161. 